\title{
Filantropía cultural y científica y reputación internacional de España. Un ejemplo de diplomacia cívica
}

\author{
Cultural and scientific philanthropy and \\ international reputation of Spain. An example \\ of civic diplomacy
}

\author{
Vicente J. Montes Gan \\ Técnico Comercial y Economista del Estado \\ Vicepresidente de la Asociación Española de Fundaciones
}

\section{Resumen}

Las diplomacias científica y cultural son hoy más importantes si cabe dada, por un lado, su capacidad contrastada para estimular la creatividad y la innovación y, por otro, su contribución a la reputación internacional como motor de diferenciación y de creación de valor. El papel de la filantropía en el fomento de la ciencia y de la cultura españolas y, por ende, de la diplomacia cívica como impulsora de las diplomacias cultural y científica, es indudable. Este trabajo analiza el lugar que ocupa España en el ámbito del mecenazgo científico y cultural, los elementos clave sobre los que cabría articular una estrategia de imagen-país en este ámbito y el resultado que cabría esperar de todo ello en relación con la imagen exterior de España.

Palabras clave: filantropía, diplomacia cívica, ciencia, cultura, reputación, imagen, España.

Clasificación JEL: Z13, Z18, O30, O38, F59.

\begin{abstract}
Scientific and cultural diplomacies are more important today, given their proven capability to stimulate creativity and innovation, as well as their contributions to international reputation as drivers of differentiation and value creation. The role of philanthropy in the promotion of Spanish science and culture, and therefore, of civic diplomacy as a vehicle of cultural and scientific diplomacy, is unquestionable. This paper analyzes the place that Spain occupies in the field of scientific and cultural patronage, the key elements on which a country-image strategy could be articulated in this area, and the return that could be expected from all of this with respect to the external image of Spain.
\end{abstract}

Keywords: philanthropy, civic diplomacy, science, culture, reputation, image, Spain .

JEL classification: Z13, Z18, O30, O38, F59. 


\section{Introducción}

La disrupción tecnológica y la conectividad global han contribuido a conformar un entorno complejo, interdependiente y altamente competitivo, en el que la reputación juega, como nunca antes, un papel relevante para los países y las organizaciones como motor de diferenciación y de creación de valor, una reputación que se fundamenta en la experiencia directa y opinión de aquellos que la perciben, así como en los mensajes que los países y sus marcas personales e institucionales comunican.

En este contexto, los dirigentes dedican sus mejores esfuerzos a establecer y reforzar aquellos elementos identitarios y atributos distintivos que pueden generar mayores retornos reputacionales a sus organizaciones. El ejercicio del gobierno y la administración de los asuntos públicos no son, ni deben ser, ajenos a esta dedicación, instrumentada a través de la diplomacia, en todas sus vertientes. Tanto en el ámbito público como en el privado se promueve la adaptación de las organizaciones a los nuevos vectores de transformación socioeconómicos a través de modelos diseñados con una narrativa para el cambio capaz de reforzar, coyunturalmente, su imagen y, estructuralmente, su reputación.

En este sentido, las diplomacias científica ${ }^{1}$ y cultural ${ }^{2}$ son hoy más importantes si cabe que en el pasado. La evidencia empírica así lo demuestra, dada su capacidad contrastada para estimular la creatividad y la innovación ${ }^{3}$. Las agencias e instituciones públicas de los ámbitos de la educación, la cultura y la investigación, las empresas y organizaciones no lucrativas, los científicos, intelectuales y artistas y las redes exteriores especializadas mediante las que se articulan, constituyen los pilares de dichas formas de diplomacia.

Entre los efectos que puede tener un esfuerzo compartido de todos estos actores en pro de la ciencia y la cultura españolas, se encuentra el fortalecimiento de los elementos intangibles que conforman nuestra marca-país, que debe entenderse como la suma de las imágenes de las marcas institucionales y personales individuales, así como articularse a través de aquellos programas que más contribuyan a potenciar dichas marcas, induciendo un impacto positivo agregado sobre nuestra reputación y la marca España.

${ }^{1}$ Nina Federoff, asesora de ciencia y tecnología de la Secretaría de Estado norteamericana, definió la diplomacia científica como «the use of scientific interactions among nations to address the common problems facing humanity and to build constructive knowledge based international partnerships» (Royal Society, 2010).

${ }^{2}$ Gienow-Hecht y Donfried (2010) señalan que la diplomacia cultural es uno de los conceptos más confusos en la historia moderna de la diplomacia. Lenczowski (2009) la define como un tipo de diplomacia pública que incluye el «intercambio de ideas, información, arte, lenguaje y otros aspectos de la cultura entre las naciones y sus pueblos con el objetivo de fomentar el entendimiento mutuo».

${ }^{3}$ A pesar de que la relación entre la ciencia y la innovación es compleja, sigue siendo evidente que la innovación, especialmente en la frontera, depende cada vez más del avance científico (OCDE, 2012). 
El papel de la filantropía en el fomento de la ciencia y de la cultura españolas y, por ende, de la diplomacia cívica ${ }^{4}$ como impulsora de las diplomacias cultural y científica, es indudable. Este trabajo incluye una visión subjetiva sobre el lugar que ocupa España en el ámbito del mecenazgo científico y cultural, los elementos clave sobre los que cabría articular una estrategia de imagen-país en este ámbito y el resultado que cabría esperar de todo ello en relación con la imagen exterior de España.

\section{Ciencia, cultura y diplomacia cívica}

El Museo del Massachusetts Institute of Technology (MIT) acogió, en el año 2018, una exposición titulada «La belleza del cerebro: los dibujos de Santiago Ramón y Cajal» organizada por el Museo de Arte Frederick R. Weisman y el Instituto Cajal del Centro Superior de Investigaciones Científicas (CSIC). Esta exposición constituyó la primera gran presentación de los dibujos pioneros que el neurocientífico español realizó del cerebro y las neuronas. Podría tratarse de una exposición más, si no fuera porque el MIT es una de las instituciones docentes e investigadoras más prestigiosas del mundo y por el reconocimiento internacional que supuso su celebración para la ciencia española.

Que los mayores expertos en neurociencia otorguen a Ramón y Cajal el carácter de fundador de la neurociencia moderna y que le sitúen, junto a Charles Darwin y Louis Pasteur, entre los tres científicos más influyentes del siglo XIX, es sin duda alguna un gran hito de la ciencia española y una acción de alto impacto de diplomacia científica con efectos directos sobre la reputación de España y su marca-país. Esta exposición fue posible gracias a la colaboración de tres fundaciones españolas, la Fundación La Caixa, la Fundación Ramón Areces y la Fundación Rafael del Pino, un ejemplo de colaboración entre instituciones científicas de diferentes países, apoyadas por organizaciones no lucrativas españolas, un caso de referencia para el estudio de la relación entre el mecenazgo y la diplomacia científica ${ }^{5}$.

El concepto de diplomacia científica ha ganado en importancia en los últimos años, un concepto que puede ser aplicado al papel de la ciencia, la tecnología y la innovación en tres ámbitos concretos: informar los objetivos y estrategias de política exterior mediante el asesoramiento científico - ciencia en la diplomacia-; facilitar la cooperación internacional en el ámbito de la ciencia -diplomacia para la ciencia-; y

${ }^{4}$ La diplomacia cívica es una forma de diplomacia ejercida por actores de la sociedad civil que representan y velan por los intereses comunes de la ciudadanía de un Estado en el ámbito internacional y que contribuyen a conformar su reputación e imagen internacional. Este tipo de diplomacia puede ejercerse de forma coordinada, en el marco de una estrategia de diplomacia pública, o de forma no jerarquizada, dada la capacidad de sus protagonistas de alinearse con el interés general sin necesidad de coordinación.

${ }^{5}$ Fue precisamente Charles Galton Darwin, nieto del afamado naturalista británico, el precursor del fenómeno de la diplomacia científica, que dio sus primeros pasos tras el final de la Segunda Guerra Mundial; en el año 1941 fue nombrado director de la Central Scientific Office británica en Washington con el mandato de colaborar con las instituciones de investigación de los Estados Unidos y facilitar el intercambio de información científica. 
usar la cooperación científica para mejorar la relaciones internacionales entre países - ciencia para la diplomacia.

De acuerdo con la Royal Society (2010), los valores científicos de la racionalidad, la transparencia y la universalidad son idénticos en todo el mundo y pueden contribuir a reforzar el buen gobierno y la confianza entre las distintas naciones: la ciencia provee un marco no ideológico para la colaboración y el libre intercambio de ideas entre personas cualesquiera que sean su cultura, nacionalidad, religión o conocimiento.

En lo que respecta al concepto de diplomacia cultural, Menéndez (2018) analiza los distintos intentos de acotar el mismo, con tres objetivos principales: la difusión de la marca-país; el intercambio y la cooperación cultural; y la promoción lingüística y educativa. Badillo (2014) engloba la diplomacia cultural dentro de un concepto más amplio, la acción cultural exterior, que desgrana combinando cuatro niveles: la acción pública exterior de los Estados en los que la cultura es la herramienta para producir atracción internacional (la diplomacia cultural); la acción pública destinada a promocionar e internacionalizar la economía cultural del país; la acción privada, encuadrada dentro de las áreas de responsabilidad corporativa de las empresas, que promueve acciones que beneficien la imagen de una marca, producto o empresa; y la promoción que el sector privado cultural hace en el exterior para favorecer sus intereses económicos. Define así la diplomacia cultural como aquella forma de comunicación exterior de los Estados en la cual la cultura desempeña una tarea central, pero instrumental, subordinada a objetivos vinculados a la imagen exterior del país. Este enfoque otorga una práctica exclusividad a los estados en la articulación de la diplomacia cultural.

Veamos otros puntos de vista sobre esta cuestión, comenzando por revisar lo que entendemos por diplomacia pública siguiendo el análisis de Sevin (2015): por un lado, Pravsak (2002) señala que la diplomacia pública es fundamentalmente un instrumento de comunicación utilizado por los Estados para alcanzar al público extranjero; por otro, los investigadores de las relaciones públicas (L'Etang, 2009) tienden a verla como una actividad dirigida por naciones, así como otras instituciones internacionales y organizaciones no gubernamentales, ejecutada mediante relaciones con los medios, actividades promocionales y estrategias persuasivas; finalmente, los estudiosos de las relaciones internacionales (Nye, 2008) llaman la atención sobre el papel de las dinámicas de poder y conceptualizan la diplomacia pública como un instrumento que los gobiernos utilizan para comunicar con y atraer al público de otros países más que meramente a sus gobiernos. No obstante, los Estados no son los únicos actores importantes en la política mundial (Yun y Toth, 2009).

En este sentido, la diplomacia cultural iría más allá de la acción exterior gubernamental. La aproximación liberal a la diplomacia pública establece que las relaciones entre Estados no pueden verse con independencia de la acción de otros referentes en el espacio internacional. En este sentido, los asuntos internacionales y políticos no son solo influenciados por los Estados, sino también por las interacciones de los actores no estatales denominados transnacionales (Keohane y Nye, 1972). Los Estados son todavía los actores dominantes y más poderosos en el ámbito internacional. Sin embargo, ya no son sus únicos protagonistas. La diplomacia pública es, por tanto, de manera creciente, una plataforma de interacción con nuevos actores no estatales. 
Como quiera que debemos entender la imagen de la cultura española como el sumatorio de las imágenes individuales de cada una de sus personalidades artísticas e intelectuales, y de sus obras y creaciones, todas las acciones realizadas por agentes públicos o privados, guiados por intereses generales o particulares, forman parte de este tipo de diplomacia, capaz de maximizar el impacto reputacional para España de cualquier acción que ponga en valor nuestra creatividad y patrimonio cultural. Este es el enfoque asumido en este documento, que destaca la importancia de la participación cívica en la acción cultural y científica de una diplomacia moderna, con una multiplicidad de actores que van mucho más allá de la intervención exclusiva de los gobiernos o la administración del Estado.

No en vano, la ciencia y la cultura son componentes clave de lo que Joseph Nye, ex decano de la Kennedy School of Government de la Universidad de Harvard, denomina soft power (Nye, 2004). La comunidad científica y cultural a menudo se encuentra en una excelente posición para solucionar problemas de interés común y apoyar formas emergentes de diplomacia que favorecen o requieren alianzas no tradicionales de naciones, sectores y organizaciones no gubernamentales.

Alineados con los objetivos de la política exterior, estos canales de intercambio entre científicos, investigadores, artistas, creadores e intelectuales se conforman como instrumentos de diplomacia científica y cultural con la capacidad de contribuir a la creación de coaliciones, a la resolución de conflictos o a la cooperación en aspectos científicos sensibles, como, por ejemplo, la no proliferación nuclear, abriendo sendas para otras formas de diálogo político (Gráfico 1).

¿Cuál es el impacto de la filantropía y, específicamente, de las fundaciones en este campo? La heterogeneidad del tercer sector y la escasez de fuentes estadísticas sectoriales sobre el mismo, que ha tratado de compensar la Asociación Española de Fundaciones con la creación del Instituto de Análisis Estratégico de Fundaciones (INAEF) ${ }^{6}$, dificulta la evaluación del impacto real de la actividad de las entidades no lucrativas en los ámbitos de la ciencia y la cultura. A pesar de ello, en los últimos años se han llevado a cabo diversos estudios que permiten aproximar el valor de dicho impacto.

Por ejemplo, el informe desarrollado a nivel europeo por la European Research Network on Philantropy (ERNOP, 2013) pone de manifiesto que, en muchos países europeos, la filantropía está siendo redescubierta como una fuente de financiación para bienes públicos e innovación social. A partir del estudio de la filantropía en 18 Estados europeos, ERNOP cifra en, al menos, 87.517 millones de euros, el valor de las contribuciones filantrópicas en Europa en el año $2013^{7}$.

${ }^{6}$ El lNAEF es una iniciativa de la Asociación Española de Fundaciones que tiene como finalidad primordial generar y difundir conocimiento sobre el sector fundacional español. El trabajo de este instituto de investigación está dando sus primeros frutos en forma de estudios específicos y publicaciones periódicas, como el Indicador de Actividad Fundacional, creado en el año 2015.

${ }^{7}$ De esta cifra, el $53 \%$ proviene de contribuciones individuales, el $25 \%$ de corporaciones y el $19 \%$ de fundaciones. España ocupa el octavo lugar entre estos países con unas contribuciones filantrópicas por un montante agregado de 3.679 millones de euros, por detrás de Alemania, Gran Bretaña, Francia, Suiza, Dinamarca, Italia y Suecia (ERNOP, 2013). 


\section{GRÁFICO 1 \\ LA DIPLOMACIA CIENTÍFICA Y CULTURAL. EL SOFT POWER DE LA CIENCIA Y LA CULTURA}

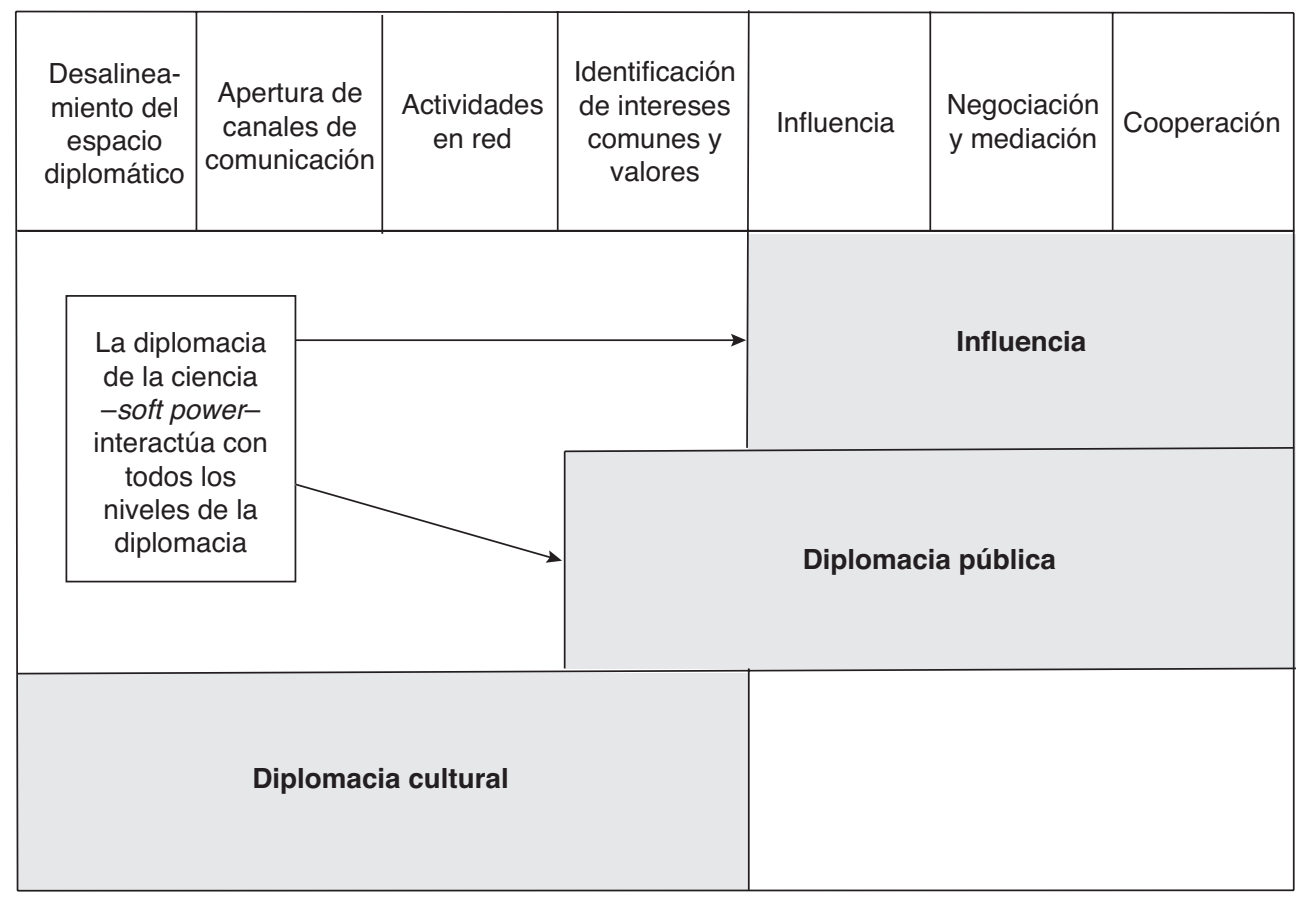

FUENTE: The Royal Society.

Por su parte, Johnson (2018) señala que en el sector no lucrativo actúan algo más de 260.000 fundaciones en los 39 países que analiza en su estudio, la mayoría de ellas situadas en Europa (60\%) y América del Norte (35\%). Sus activos totales alcanzan 1,5 billones de dólares ${ }^{8}$ y su gasto anual supera los 150.000 millones de dólares.

Ninguno de los estudios mencionados recoge en su integridad la dimensión real del sector no lucrativo, pero nos permiten vislumbrar la capacidad de acción de estas instituciones en la actualidad. Es importante tomar en consideración que se trata de un sector de desarrollo reciente, en fase de crecimiento, en el que el $72 \%$ de las fundaciones fueron creadas durante los últimos 25 años.

8 Estados Unidos, cuyas fundaciones gestionan unos activos de 890.000 millones de dólares, lidera este ranking. Las fundaciones holandesas, segundo país en el ranking, administran unos activos de 108.000 millones de dólares. Y las de Alemania, que ocupa el tercer lugar, cuentan con 92.900 millones de dólares. España ocupa la octava posición en el ranking con un total de 29.000 millones de dólares en activos, justo por detrás de Francia y por encima de países como China, Australia o Canadá. 


\section{Mecenazgo y diplomacia científica}

A fin de lograr el máximo desarrollo de la ciencia y la innovación y, con ello, materializar el objeto y contenido de la diplomacia científica, resulta determinante tomar en consideración la actividad filantrópica y el mecenazgo, en sus distintas vertientes. Se trata de un mecenazgo ejercido desde la sociedad civil, de forma operativa, a través de las empresas y de las organizaciones no lucrativas. Este trabajo centra su atención en el papel de las segundas en el fomento de este tipo de diplomacia.

Como señala la Royal Society (2010), las organizaciones científicas, incluidas las academias nacionales, juegan un papel muy importante en la diplomacia científica, especialmente cuando las relaciones formales políticas entre países son débiles. La comunidad científica es capaz de liderar nuevas y diferentes formas de colaboración internacional y, para lograrlo, el papel de las organizaciones no lucrativas, las agencias multilaterales y otras redes informales es muy relevante.

En el apartado anterior se ha mencionado la dimensión estimada del fenómeno de la filantropía, pero, en lo que respecta, específicamente, al apoyo a la investigación y la innovación, la Comisión Europea ha desarrollado el estudio EUFORI (Gouwenberg et al., 2015) cuyo objetivo es dimensionar el apoyo de las fundaciones europeas a las iniciativas de investigación e innovación. Este estudio toma en consideración una muestra de 12.941 fundaciones de 27 países europeos con inversiones en actividades de investigación e innovación. En el estudio final participaron las 1.591 fundaciones más importantes en este campo. La principal conclusión fue cifrar el presupuesto total anual aplicado por las mismas a estas actividades en 5.000 millones de euros, un montante que supone, aproximadamente, la mitad del presupuesto medio anual que la Unión Europea destinará a los investigadores durante la duración del Programa Horizonte 2020, cifra que da una idea de la importancia de la actividad de las fundaciones en este campo'.

El informe sobre España de EUFORI fue elaborado por los profesores Marta Rey y Luis Ignacio Álvarez (2015). En este estudio, de las 458 fundaciones de investigación e innovación a las que se envió el cuestionario, respondieron 229. Los resultados del estudio caracterizan a este sector fundacional como relativamente joven, dinámico y diverso, con un ámbito de actuación no solo local o regional sino también nacional. Las fundaciones analizadas sumaban más de $4.690 \mathrm{mi}-$ llones de euros en activos, 980 millones de euros en ingresos y 773 en gastos en proyectos de investigación e innovación en el año 2012. Entre estas fundaciones, el $70 \%$ declaró desarrollar proyectos de investigación básica y el $94 \%$ de investigación aplicada.

${ }^{9} \mathrm{El}$ origen de los recursos de las fundaciones incluidas en el Informe EUFORI proviene en un 54\% de donantes privados individuales y familias, en un $18 \%$ de corporaciones empresariales, en un $18 \%$ de organizaciones no lucrativas y en un $17 \%$ del sector público. El $81 \%$ de estas fundaciones apoya proyectos de investigación aplicada mientras que el $61 \%$ de ellas apoya, también, proyectos de investigación básica (Gouwenberg et al., 2015). 
Para un mayor detalle sobre las actividades de las fundaciones españolas en este campo, cabe recurrir a dos fuentes principales: por un lado, el Consejo de fundaciones para las ciencia, que integra a las principales fundaciones dedicadas al apoyo a la investigación y la innovación bajo la coordinación de la Fundación Epañola para la Ciencia y la Tecnología (FECYT), y que publica un catálogo de ayudas para la investigación e innovación; por otro lado, la Asociación Española de Fundaciones creó el Grupo Sectorial de Fundaciones de la Salud, Investigación y Bienestar, en el que se realizan análisis sectoriales.

La motivación principal de las organizaciones no lucrativas para apoyar la investigación básica es que su financiación suele darse en un horizonte temporal largo y conllevar riesgos de ingresos inciertos. En este escenario, su apoyo es determinante ya que, aunque las empresas invierten en investigación básica, ésta aún depende de la investigación pública y de los organismos no lucrativos. En los países de la OCDE, alrededor del $58 \%$ de la investigación básica se lleva a cabo en el sector de la educación superior y un $22 \%$ en el sector de gobierno. En España el $60 \%$ de la investigación básica se lleva a cabo en las universidades (OCDE, 2012).

Un caso aparte es el fenómeno de la filantropía orientada al desarrollo, que también formaría parte de la lógica de la diplomacia científica. La OCDE a través de su Centro de Desarrollo ha calculado la cantidad total desembolsada para proyectos de desarrollo por las organizaciones no lucrativas en los 8.000 millones de dólares anuales de media en el trienio 2013-2015, lo que significa una cifra total de 23.900 millones. Aunque los fondos filantrópicos son solo una porción del total de fondos aportados a un proyecto, la influencia que ejerce en los donantes privados es mayor, en muchos casos, a su aportación. La salud es un ejemplo de cómo un donante focalizado, estratégico y persistente puede arrastrar al conjunto de la comunidad de donantes e incluso a instituciones multilaterales tan poderosas como la Organización Mundial de la Salud. Por ello, la filantropía privada está llamada a jugar un papel cada vez más relevante en la hoja de ruta del desarrollo durante los próximos años. Los Objetivos de Desarrollo Sostenible (ODS), referencia indiscutible para la diplomacia científica, responden a un juego de condiciones necesarias en el que el sector privado realiza una contribución imprescindible en forma de recursos económicos, facilitación política y creatividad. Para que esto ocurra es necesario fortalecer la coordinación entre los distintos actores, consolidar marcos legales favorables y favorecer la transparencia sobre medios y resultados (OCDE, 2017; Fanjul, 2018).

\section{Filantropía y diplomacia cultural}

La actividad de las fundaciones españolas ha estado muy unida al sector cultural en España. Se trata de un sector importante de nuestra economía, con un gran potencial de crecimiento, que integra un conjunto de actividades, bienes y servicios, relevantes en términos económicos, pero que incorporan, además, elementos intangibles 
que contribuyen al reforzamiento del atractivo de España y de «lo español» y de su reputación internacional.

La contribución de las industrias culturales y creativas al producto interior bruto y al empleo en España alcanza el 6,9\% y el 5,2\%, respectivamente (Deloitte, 2017). Existen 118.407 empresas culturales que emplean a 687.200 personas, un 3,7\% del empleo total, y atienden un mercado interior de bienes y servicios culturales de 13.298 millones de euros, cifra que representa el 2,5\% del gasto total estimado en bienes y servicios en España (Ministerio de Cultura y Deporte, 2018).

En perspectiva internacional, el Anuario de Estadísticas Culturales señala que el valor de las exportaciones de los bienes vinculados a la cultura se situó, en el año 2017, en 1.933 millones de euros, y las importaciones, en 2.319 millones de euros. Las entradas de turistas internacionales por motivos culturales alcanzaron los 12,8 millones en ese año, el 18,1\% del total de viajes realizados a España por ocio, recreo o vacaciones, y generaron unos ingresos de 13.924 millones de euros. El atractivo internacional de la cultura española tiene que ver con su ingente patrimonio cultural, que incluye 17.579 bienes de interés cultural, pero también con la calidad y heterogeneidad de su oferta actual de bienes y servicios culturales.

De acuerdo con Reputation Institute (2018), España ocupa la novena posición del Country RepTrak 2018 por su contribución a la cultura global ${ }^{10}$, en un ranking que lideran los países europeos, con Italia a la cabeza, seguida de Francia, Reino Unido, Japón y Suecia. Se trata de una buena, aunque mejorable, posición.

Tanto en la conservación y promoción del patrimonio cultural como en el apoyo a la creación y difusión de nuestros bienes y servicios culturales, el papel de las fundaciones españolas ha sido y es muy relevante. De hecho, un estudio reciente, impulsado por la Asociación Española de Fundaciones (Fernández y Gross, 2018), señala que la principal dedicación de las fundaciones es la actividad cultural, seguida de la educación e investigación.

El Gráfico 2 muestra con claridad el sesgo hacia estas actividades de las fundaciones españolas. En concreto, el 39\% de la actividad de las mismas se centra en el ámbito de la cultura, un porcentaje que aumenta hasta el $46 \%$ en el caso de las fundaciones filantrópicas personales y familiares (FFPF) ${ }^{11}$.

Estos datos permiten atisbar la aportación actual de las fundaciones privadas, en especial de las FFPF y de las fundaciones empresariales, en el campo de la cultura, así como el impacto de su actividad, actual y futura, en la imagen y reputación de España.

${ }_{10}$ Reputation Institute pregunta a una muestra de público general de 28 países sobre sus percepciones tanto de su propio país como de otros. Acerca de la cultura, plantea si el país contribuye de forma considerable a la cultura global, si en él nacieron muchos artistas, científicos, inventores, escritores, deportistas y políticos muy conocidos.

${ }^{11}$ El grupo de FFPF está constituido por todas aquellas entidades cuyo patrimonio procede de una misma persona o familia y cuya gestión les permite actuar autónomamente en beneficio de la sociedad. La exhaustiva tarea de identificación de las fundaciones que cumplen este criterio, entre la totalidad de las fundaciones españolas, realizada por Fernández y Gross (2018), permite cuantificar este colectivo en 1.635 entidades, cifra que representa el 18,5\% del total de fundaciones activas. 


\section{GRÁFICO 2}

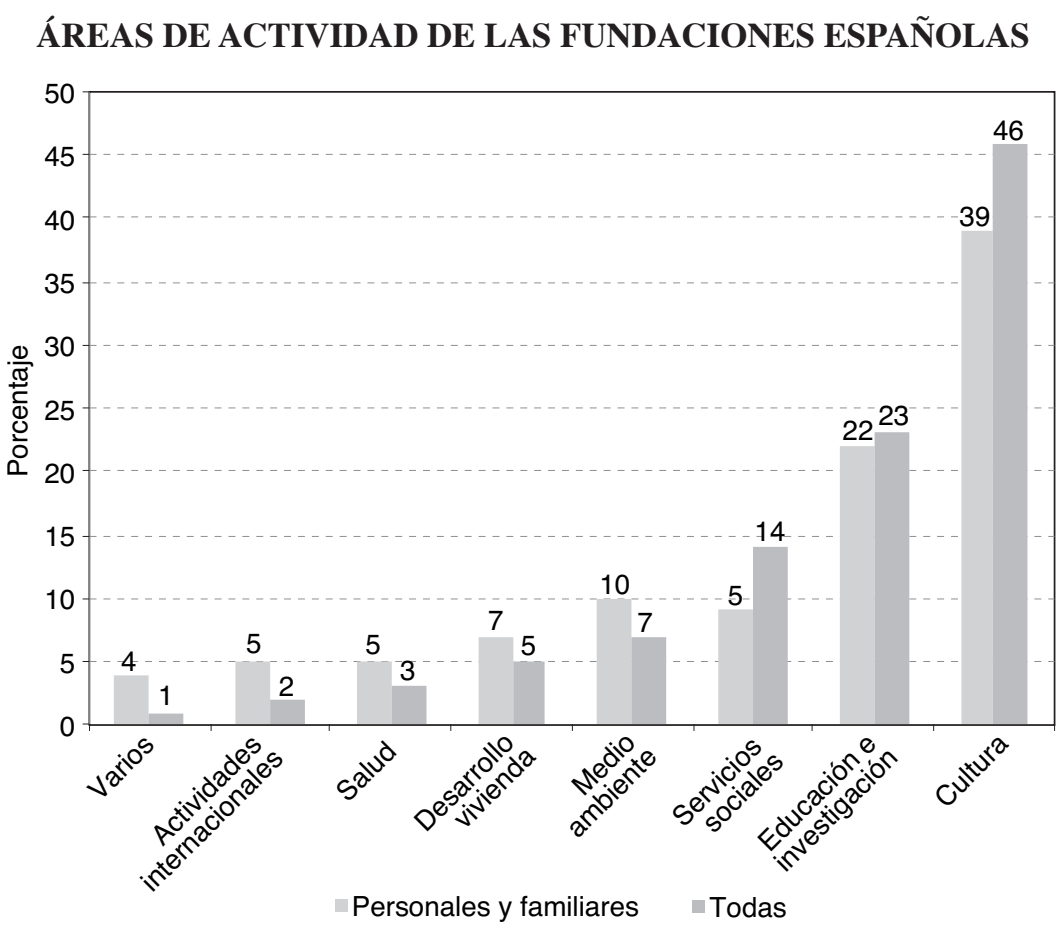

FUENTE: Elaboración propia

En este sentido, merecen una mención especial las actividades de las fundaciones de los grandes grupos de comunicación privados españoles. No en vano, la televisión continúa siendo el principal canal para crear imagen país en el mundo ${ }^{12}$. La internacionalización de estos grupos de comunicación y su intensa labor de creación de contenidos y de desarrollo de proyectos culturales de alta calidad ${ }^{13}$, les convierten en actores clave de la diplomacia cultural española.

Deloitte (2017) muestra el impacto de la televisión en abierto en el impulso de la cultura y de la reputación de España en el mundo. Los operadores de televisión en abierto financiaron, en el año 2015, el $68 \%$ de las obras audiovisuales europeas en España y constituyeron, además, el motor de la cultura y la diversidad lingüística, además de impulsar la marca España, que se situó en el cuarto lugar del mundo en la comercialización internacional de formatos televisivos.

12 Canalizada de forma creciente a través de las redes sociales. En este sentido Marco y Otero (2010) adelantaban hace una década que las industrias culturales se basaban en la distribución del proceso creativo para generar riqueza «las ideas se convertían en un producto distribuido después a través de la industria musical, cinematográfica, editorial», pero que las nuevas formas de acceso a la cultura han modificado este esquema al introducir la variable social.

${ }^{13}$ El $93 \%$ de la financiación de obras europeas realizada por los operadores de televisión en abierto en España en el año 2015 se destinó a producciones españolas (Deloitte, 2017). 
La estrategia pública de actuación de España en materia de diplomacia cultural fue diseñada a finales de los años 80 y principios de los 90 sobre la base de cuatro pilares: idioma, Iberoamérica, Europa y la Unesco. Esta estrategia ponía el acento en el idioma español, aprovechaba la posición ventajosa que en aquellos momentos tenía España como líder de las culturas del español y del portugués, hacía valer nuestra condición de enlace con las instituciones europeas y asumía la filosofía de la Unesco, que priorizaba la cooperación y la diversidad cultural. En las tres décadas transcurridas desde entonces, se han cumplido muchos de sus objetivos. Entre ellos, destaca la consolidación del Instituto Cervantes, programas de cooperación como Ibermedia, la Asociación de Academias de la Lengua, etc. (Álvarez, 2019); en estas y en otras iniciativas de diplomacia cultural, las organizaciones no lucrativas tuvieron un papel muy relevante.

La actual arquitectura gubernamental de diplomacia cultural, liderada por los departamentos de asuntos exteriores, cultura y comercio, incluye: en las Embajadas de España, 120 Consejerías Culturales, a las que se suman 98 Oficinas Económicas y Comerciales y 31 Oficinas Técnicas de Cooperación Internacional; 87 delegaciones del Instituto Cervantes; 19 Centros Culturales, así como Institutos culturales autonómicos (Etxepare, Institut Ramon Llull, etc.); la Red de Casas (Casa Asia, Casa Árabe, Casa Sefarad, Casa África y Casa Mediterránea); Acción Cultural Española AC/E ${ }^{14}$; y otros organismos con actividades en este campo, como por ejemplo, la Fundación Carolina, la Fundación Onuart o el ICEX España Exportación e Inversiones.

La dimensión del sector fundacional y su intensa actividad en el campo de la cultura dan idea del potencial de estas instituciones para contribuir a una estrategia compartida de diplomacia cultural. De acuerdo con Dafne (2019), el sector fundacional español incluye 8.866 entidades operativas con 256.867 empleados y un conjunto de activos valorados en 21.469 millones de euros. Estas instituciones realizan cada año una inversión de 8.066 millones de euros en actividades filantrópicas ${ }^{15}$.

Estas cifras dan idea del valor que puede añadir a la estrategia actual de diplomacia cultural una mayor colaboración entre el ámbito público y privado ${ }^{16}$, en una relación que, para ser posible, debe partir de un diálogo basado en el respeto mutuo. Hay que tomar en consideración que las fundaciones son, en su mayoría, operativas

14 Acción Cultural Española AC/E declaró en 2017 una inversión de 7,7 millones de euros en actividades de difusión de la cultura española.

15 Estas cifras contrastan con el uso limitado por parte de la ciudadanía española del sistema de incentivos fiscales por inversiones y gastos de interés cultural introducido en nuestra legislación en los ámbitos estatal y autonómico y, en particular, de las deducciones en el IRPF. Rubio y Villarroya (2019) analizan estas cuestiones y realizan propuestas interesantes para aumentar la participación cívica en este campo.

${ }^{16}$ En este sentido, resulta interesante analizar otros modelos de diplomacia cultural, con sus aciertos y sus errores. Francia, por ejemplo, dispone de dos redes complementarias en este campo, una de carácter público, articulada en torno al Institut Français, y otra privada, las Alliances Françaises. Estas últimas constituyen franquicias privadas independientes y autofinanciadas en un $96 \%$ a través de la enseñanza del idioma francés y la emisión de certificados (KEA, 2016). La Fondation Alliance Française establece los criterios comunes y estudia la viabilidad de cada nueva Alliance Française propuesta. Otro ejemplo interesante es China, cuyos Institutos Confucio, que ya superan el medio millar en el mundo, se organizan mediante una fórmula de franquicia privada que permite que, incluso, coexistan varios centros en la misma ciudad. 
y que tienen misiones establecidas a priori, así como un sólido sistema de rendición de cuentas. Álvarez (2019) señala que el éxito de la diplomacia cultural depende en gran medida de que sus contenidos sean conocidos y asumidos por todos los actores no gubernamentales del país que participan, de alguna manera, en las relaciones internacionales y que debe prevalecer la mayor coherencia posible entre la cultura que se hace viajar y la cultura que se promueve por medio de las políticas internas.

En esta línea, Marco y Otero (2010) señalan que el papel de la diplomacia cultural es clave para convertir los activos culturales en soft power, no solo por medio de sus funciones tradicionales de fomento de las actividades culturales en el exterior, sino mediante el desarrollo de una colaboración más estrecha con el sector privado, tanto para potenciar la utilidad social de las alianzas público-privadas en el plano internacional como para fomentar la internacionalización de las empresas culturales.

\section{Ciencia, cultura, reputación y e-comunicación}

Nunca antes en la historia de la humanidad tuvimos la oportunidad, como hoy, de compartir globalmente el conocimiento humano, ni el cambio en multitud de ámbitos adquirió un carácter tan exponencial (Rifkin, 2011 y 2014). Ese cambio también se ha producido en la articulación de los mensajes y ha modificado el paradigma de la comunicación. Internet ha universalizado el lenguaje multimedia y ha trastocado nuestra comprensión de los contenidos informativos y de los procesos de creación de mensajes que, además, convergen de manera creciente hacia entornos colaborativos reforzados por las innovaciones de la sociedad digital.

Hoy los ciudadanos pueden acceder de forma directa a la información sin la mediación de los medios de comunicación profesionales y pueden publicar informaciones en plataformas de seguimiento masivo al margen de los editores de los medios tradicionales. La que podríamos denominar, e-comunicación, ha cambiado la metodología de creación de mensajes y, con ello, ha modificado la forma de conformar los atributos de imagen que fraguan en la reputación de las personalidades individuales, las instituciones y organizaciones y, a nivel agregado, de las naciones.

En el contexto de este cambio de paradigma de comunicación, durante la última década, el concepto de marca-país ha atraído el interés de académicos y gobiernos, impulsados por la creencia de que una marca nacional fuerte puede contribuir al desarrollo sostenible (Jaffe y Nebenzahl, 2001), restaurar la credibilidad internacional, reforzar la influencia política global y fortalecer las relaciones y la cooperación con otras naciones (Yan, 2003).

La marca-país ha sido analizada en la literatura de la mercadotecnia desde principios de la década de los años noventa. Helmi y Mulyanegara (2011) señalan a Kotler et. al. (1993) como los primeros académicos que discutieron estas cuestiones y, en concreto, la importancia de la marca-país para atraer la inversión exterior y el turismo internacional. Asimismo, destacan que una visión general de la literatura en este campo sugiere que hay cuatro ámbitos principales que sustentan los fundamentos 
teóricos de la marca de una nación: el país de origen (Parameswaran y Pisharodi, 1992; Roth y Romeo, 1992; Shimp, Saeed y Madden, 1993); la imagen de producto-país (Martin y Eroglu, 1993; Parameswaran y Yaprak, 1987); la marca de destino (Hankinson, 2007; Pritchard y Morgan, 1998); y la identidad del país (Anholt, 2007; Keillor y Hult, 1999; Keillor, Hult, Erffmeyer y Babakus, 1996). Aunque la mayoría de los debates sobre la marca-país tuvieron lugar en el marco de la literatura del marketing, en los últimos años ha quedado claro que la cuestión es mucho más amplia ya que involucra a distintas disciplinas, como las relaciones internacionales, la ciencia política o la diplomacia pública (Anholt, 2003), entre otras.

A pesar del creciente número de investigaciones orientadas durante la última década al estudio de la marca nacional, muy pocos autores en la literatura del marketing han centrado su mirada, específicamente, en el papel de las entidades no lucrativas en este campo. Quizás el primer estudio centrado en la relación entre la marca-país y el desarrollo de actividades filantrópicas y del mecenazgo fue el desarrollado en el año 2011 por Jessica Helmi y Riza Casidy Mulyanegara, profesores de la Deakin University (Melbourne, Australia). Helmi y Mulyanegara (2011) analizan estas cuestiones y desarrollan un marco conceptual para comprender el comportamiento de los mecenas frente a una percepción de marca-país; esta se conforma como una variable mediadora entre los motivos de la donación y el comportamiento del donante. Su análisis concluye que la percepción sobre la marca-país tiene implicaciones significativas en lo que respecta a la disposición y el alcance de la contribución a una causa benéfica asociada con la nación. La imagen de marca de las organizaciones benéficas puede moderar la relación entre la percepción de la marca del país y el comportamiento del donante. En el estudio también se señala que las comunicaciones de marketing integradas afectan significativamente a la percepción de las marcas nacionales y a la imagen de marca de las organizaciones no lucrativas.

La marca-país refleja la conjunción de los elementos negativos y positivos que caracterizan y distinguen a un determinado país en el escenario internacional. Integra las imágenes de marca e identidades individuales junto a otros elementos de diversa índole: geográficos, sociales, políticos, históricos, institucionales, científicos, tecnológicos, etc. Esta variedad y heterogeneidad de atributos que incorpora la imagen-país impide que la misma pueda ser monopolizada (Anholt, 2005). En lo que respecta a la imagen de la ciencia-país o la cultura-país, el espectro de sujetos que contribuyen a definirla incluye a las universidades y centros de investigación, las empresas, los filántropos y entidades del tercer sector, las personalidades destacadas de la sociedad en el campo de la ciencia y la cultura-divulgadores, académicos, dramaturgos, artistas, científicos, altos funcionarios, empresarios, etc.-; pero también a los ciudadanos, al viajar y relacionarse fuera del país o dentro del mismo, a los medios de comunicación, la Administración pública en todos sus niveles, etc.

Una buena marca-país contagia los bienes y servicios producidos en el mismo, reforzando su valor, y atrae, no solo a consumidores o turistas, sino también a inversionistas y mecenas. De hecho, la imagen y el prestigio de un país están interrelacionados: la imagen es la identidad que se proyecta al mundo; el prestigio, la 
información recibida dada por la experiencia del visitante, o inversionista (Whetten y Mackey, 2002) y también, de los filántropos.

En el ámbito científico y cultural no es muy distinto, la imagen de la ciencia-país y de la cultura-país otorga prestigio y credibilidad a nuestros creadores e investigadores, a los centros culturales y de investigación y a las instituciones privadas y públicas que las fomentan, así como a los proyectos empresariales e institucionales innovadores, afectando primero a la propia ciencia y cultura españolas y, a través de ellas, a la marca-país.

Además, en el caso específico de la ciencia, la distinción entre mensaje y contexto es más relevante si cabe que en el de otros componentes de la imagen-país. Resulta relativamente sencillo, aunque costoso, definir un mensaje y desarrollar una campaña de comunicación internacional sobre los valores de la ciencia española, pero es muy difícil cambiar el contexto en el que los mensajes se perciben y hacer sostenible ese mensaje si el mismo no va acompañado de resultados que sustenten los argumentos empleados. La reputación en este campo se basa, sobre todo, en los resultados.

De hecho, los medios de comunicación internacionales reaccionan de manera diferente ante las noticias provenientes de países con una tradición-marca científica más o menos consolidada: actúan con indiferencia ante los éxitos de los países con una marca débil; mientras que los países con marcas fuertes tienen pocos problemas para conseguir la atención de sus realidades científicas más mediocres.

\section{Reforzamiento de la estrategia de marca-país a través del mecenazgo de la ciencia y la cultura}

Como se ha señalado, en la actualidad, las organizaciones no lucrativas españolas desarrollan un gran número de actividades de apoyo a la cultura y a la ciencia, así como de fomento de la creación y la investigación, con estrechos vínculos con lo que conocemos como diplomacia científica y cultural.

En sus distintas vertientes de, ciencia y cultura en la diplomacia, diplomacia para la ciencia y la cultura, y ciencia y cultura para la diplomacia, las actividades mencionadas en los apartados anteriores muestran la importancia de las actividades de estas organizaciones y su aportación a la imagen y reputación de nuestra ciencia y cultura en el mundo y, a través de ella, de nuestra marca-país. No obstante, el sector no lucrativo español no ha desarrollado todo su potencial en este campo y una adecuada parrilla de incentivos, incluyendo una regulación eficiente del mismo, podría favorecer su expansión con un impacto muy positivo en el reforzamiento de la creatividad y la innovación en España.

Con el objetivo de impulsar y aumentar las iniciativas de mecenazgo y su articulación operativa, habría que comenzar por contribuir a prestigiar la filantropía en la sociedad española. Es necesario inducir un cambio de percepción en la misma a través de la educación, así como comprender los entornos de comunicación en los que se conforman los mensajes negativos que se materializan en un daño reputacional desincentivador de las iniciativas filantrópicas, y actuar en consecuencia. 
No obstante, sin perjuicio de la importancia de prestigiar la filantropía y de dar una mayor visibilidad a las organizaciones no lucrativas, quizás la prioridad para las Administraciones públicas debe ser la articulación de una regulación incentivadora de una mayor participación cívica ${ }^{17}$. Se trata de fomentar la filantropía y el mecenazgo como práctica habitual en la sociedad, tanto por parte de empresas y fundaciones, como por parte de la sociedad civil en su conjunto.

La ciencia española merece un tratamiento especial y prioritario, dado el peor posicionamiento reputacional de la misma a nivel internacional en plena revolución industrial y tecnológica. A diferencia de lo ocurrido en el caso de la cultura, de acuerdo con Reputation Institute (2018), España obtiene en ciencia-innovación una percepción moderada. En términos comparativos, España alcanza uno de los peores resultados del ranking, pues ocupa la vigesimocuarta posición. En el contexto de la Unión Europea se sitúa solo por delante de Portugal, República Checa, Polonia, Grecia y Rumanía ${ }^{18}$.

A fin de favorecer el mencionado cambio de cultura, se debe profundizar en el conocimiento de las relaciones entre ciencia, tecnología y sociedad, así como analizar la percepción de la ciudadanía, a nivel internacional, sobre los avances científicos y tecnológicos, y sobre la capacidad de éstos para la mejora de la calidad de vida de la población.

¿Qué hacer desde la filantropía para impulsar la reputación de la ciencia española en el mundo? (Cuadro 1). El apoyo de la filantropía a la «ciencia en la diplomacia», puede materializarse por muy distintas sendas, por ejemplo, contribuyendo a reforzar los mensajes sobre el valor de la ciencia en los ministerios implicados en la acción exterior y en las embajadas, incorporando el estudio de la ciencia y de las políticas científicas en los programas de formación continua de los equipos directivos de aquellos ministerios, integrando, incluso, a un mayor número de científicos en las plantillas de los mismos con funciones de asesoramiento a los perfiles senior en los niveles estratégicos.

Asimismo, más allá de la labor realizada por las instituciones públicas sectoriales, cabría ampliar la selección de graduados en estudios de ciencia para su integración como parte del personal diplomático, impulsando además la formación continua de éstos en instituciones científicas ${ }^{19}$ y el desarrollo de organizaciones científicas independientes que provean a los ministerios de acción exterior de informes sobre política de ciencia ${ }^{20}$.

${ }^{17}$ Las dificultades para alcanzar los consensos necesarios para impulsar una Ley de mecenazgo o la regulación ineficiente de actividades como el crowdfunding son muestras claras del desinterés de los poderes públicos en España por el fomento de la filantropía, el mecenazgo y la participación cívica en general.

18 Sobre los atributos de tecnología-innovación, Reputation Institute (2018) pregunta a los encuestados si el país es tecnológicamente avanzado, así como si se encuentra entre los primeros países en crear y comercializar nuevas tecnologías.

19 Un ejemplo de ello en el campo de las ciencias sociales es la Fellowship Rafael del Pino-MAEC en la Harvard Kennedy School of Government resultado de la cooperación de la Fundación Rafael del Pino y el Ministerio de Asuntos Exteriores y de Cooperación Internacional.

${ }^{20}$ Entre ellas, el Consejo de Fundaciones para la Ciencia o el Grupo Sectorial de Fundaciones de la Salud, Investigación y Bienestar de la Asociación Española de Fundaciones. 


\section{CUADRO 1 \\ PROPUESTAS DESDE LA FILANTROPÍA PARA LA PROMOCIÓN DE LA CIENCIA Y LA CULTURA ESPAÑOLAS Y LA MEJORA DE SU REPUTACIÓN INTERNACIONAL}

1. Incluir dentro de las estrategias de marca-país a las organizaciones de la sociedad civil y, muy en particular, a las fundaciones, en pie de igualdad con la administración pública, las empresas y otros agentes.

2. Favorecer la visibilidad de las fundaciones y de los mecenas que apoyan la creatividad y la innovación, así como contribuir a la mejora de la percepción social de la actividad de las fundaciones y de la iniciativa de sus fundadores.

3. Conformar un marco regulatorio incentivador del mecenazgo y de la creación, dinamismo y capacidad operativa de las fundaciones que apoyan la ciencia y la cultura y, específicamente, eliminar la legislación discriminatoria actual, que desincentiva el apoyo de las instituciones no lucrativas a la presencia de investigadores y creadores españoles en los principales centros internacionales de excelencia científica y cultural.

4. Establecer un marco de colaboración entre la Administración pública y la Asociación Española de Fundaciones, en el contexto de la contribución de las mismas a las diplomacias científica y cultural, muy en particular en lo que respecta al apoyo a las redes independientes de científicos y creadores españoles en el exterior.

5. Asegurar un mayor conocimiento entre los dirigentes del servicio diplomático nacional de la realidad actual de la ciencia y la cultura españolas y del papel de las fundaciones en su promoción y difusión.

6. Conformar sendas estrategias de marca «Ciencia española»y «Cultura española» alineadas con los grandes compromisos sociales universales, por ejemplo, los Objetivos de Desarrollo Sostenible de Naciones Unidas, sustentadas en la figura de los embajadores honorarios de la ciencia y la cultura españolas.

7. Apoyar firmemente desde la Administración pública las iniciativas independientes de la sociedad civil dirigidas a promover el desarrollo de la ciencia y la cultura españolas, canalizadas, muchas de ellas, a través de la actividad o con el liderazgo, de las fundaciones, asumiendo una metología de acompañamiento orientada a la obtención del mayor retorno reputacional.

8. Centrar la atención, a este respecto, en los científicos y creadores, entendiendo las marcas «Ciencia española»y «Cultura española» no como realidades en sí mismas, sino como la suma de las imágenes individuales de cada de nuestros científicos y creadores, concentrando los esfuerzos desde la Administración pública en potenciar a los mismos individualmente, conformando nuestra imagen-país como sumatorio de las imágenes individuales de cada uno de ellos.

Es necesario establecer mecanismos y espacios para el diálogo más efectivos entre los actores de las políticas públicas, los académicos, los investigadores y la filantropía, trabajando en una política exterior capaz de identificar proyectos y procesos que puedan interesar tanto a los diplomáticos como a los científicos. 
Las Fundaciones pueden asumir un papel relevante como catalizadores de ese diálogo. Un ejemplo es el debate sobre la Agenda 2030 y los Objetivos de Desarrollo Sostenibles (ODS) aprobados por la Asamblea General de las Naciones Unidas. Las organizaciones no lucrativas y las empresas ${ }^{21}$ contribuyeron, en gran manera, a impulsar el debate sobre el papel de la innovación en el logro de estos objetivos ${ }^{22}$, un aspecto de enorme transcendencia política a nivel mundial. El alineamiento de todos los actores relevantes entorno a los ODS permitiría a España fraguar un potente mensaje de la ciencia española, como fuente de soluciones innovadoras para los principales problemas de la humanidad, y con efectos positivos sobre los mejores atributos reputacionales de España.

Acerca de la «diplomacia para la ciencia», las organizaciones no lucrativas concentran sus esfuerzos en este campo y son claves para facilitar la cooperación internacional en el ámbito de la ciencia. Fundaciones como La Caixa, Botín, Ramón Areces, Barrié de la Maza o Rafael del Pino, entre otras, becan cada año a casi un millar de investigadores para el desarrollo de sus proyectos en los mejores destinos científicos del mundo. Este hecho ha llevado a estas y otras fundaciones a establecer alianzas con estos centros de excelencia, una vinculación institucional que está teniendo un retorno evidente en términos de la valoración internacional de la ciencia española y, con ello, en la marca España.

Quizás sea en el ámbito de la «ciencia para la diplomacia» en el que el papel de las organizaciones no lucrativas es más débil en la actualidad. El caso de Santiago Ramón y Cajal mencionado anteriormente, puede ser un referente de la colaboración en este tipo de diplomacia. A este respecto, y con el objetivo de utilizar la cooperación científica para mejorar la relaciones internacionales de España, cabe ahondar en el nombramiento de embajadores de la ciencia española, tanto actuales como de todos los tiempos, permitiendo la articulación de una política de divulgación y difusión de los avances de la ciencia española ya sea a través de los grandes actores en la historia de la ciencia, como Santiago Ramón y Cajal, o de los investigadores y científicos españoles actuales, entendiendo la imagen de la ciencia española como la suma de las imágenes individuales de todos ellos, contribuyendo así a conformar una marca de la ciencia española más potente ${ }^{23}$.

En este sentido, la articulación de la diáspora científica española debe ser una prioridad. Aunque la ciencia no tenga patria sí que la tienen los científicos españoles residentes en el exterior, agrupados de manera creciente en redes independientes.

\footnotetext{
${ }^{21}$ A través de sus departamentos de responsabilidad social y de sus fundaciones corporativas.

${ }^{22}$ En el año 2015 la Fundación Rafael del Pino acogió los encuentros organizados por la Red española del Pacto Mundial de Naciones Unidas que permitieron materializar el punto de vista del sector privado en el debate y la negociación de los ODS. Estos encuentros fraguaron en un documento estratégico basado en las experiencias de las empresas, la Administración Pública, el tercer sector y las instituciones educativas en este campo. Más recientemente, las mismas instituciones han puesto en marcha la iniciativa GoODS con el objetivo de difundir y reconocer la innovación orientada al logro de los ODS.

${ }^{23}$ La Fundación Española para la Ciencia y la Tecnología (FECYT) y la Oficina para Asuntos Culturales y Científicos de la Embajada de España en Londres, con la colaboración de la Sociedad de Científicos Españoles en el Reino Unido (SRUK/CERU) organizan encuentros bajo el apelativo «Embajadores para la ciencia».
} 
Por ejemplo, las fundaciones patrimoniales, como representantes independientes de la sociedad civil, han demostrado una mayor facilidad que el gobierno y las administraciones públicas para contribuir a impulsar determinadas redes de profesionales españoles en el exterior. Estas redes son claves para defender sus intereses, así como para la articulación internacional de los mismos, y son colaboradoras necesarias para una estrategia compartida de marca-país ${ }^{24}$.

Muchas de estas ideas son extrapolables al ámbito de la cultura, entendida en su triple vertiente de cultura en la diplomacia, diplomacia para la cultura y cultura para la diplomacia.

El Cuadro I recoge un conjunto de recomendaciones centradas en los ámbitos de la ciencia y de la cultura, con el objetivo de favorecer el aumento de la aportación del tercer sector español a la imagen y reputación de nuestra ciencia y cultura en el mundo y, a través de ella, de la marca España.

Otra cuestión recomendable sería introducir un mayor número de fundaciones entre los actores principales de la estrategia de marca-país. Las fundaciones y otras entidades sin ánimo de lucro deben ser invitadas a integrarse en este esfuerzo compartido. Para ello debe reconocerse a las mismas su importancia en este campo y perfilar un eje estratégico adicional que permita alinear la actividad de estas con las políticas públicas, y viceversa, lo que no es menos importante, con sinergias evidentes para la diplomacia científica y cultural y la articulación de las distintas diásporas, artística, investigadora, emprendedora, empresarial, profesional, etc.

Pensemos que, aunque generalmente muchas de las fundaciones centran su atención en el apoyo, en exclusiva, a los ciudadanos españoles, como se ha señalado, la apuesta por la excelencia las lleva a desarrollar una intensa actividad en los centros educativos e investigadores más relevantes del mundo, un espacio en el que el impacto de nuestros profesionales induce un efecto multiplicador de marca-país muy significativo.

Es el caso, por ejemplo, de los programas de becas para estudios de posgrado impulsados por las fundaciones, que permiten a los estudiantes y profesionales españoles más brillantes desarrollar sus actividades formativas, docentes, creativas o investigadoras en centros que por su relevancia son el mejor altavoz sobre la excelencia de nuestros conciudadanos, con retornos evidentes en términos de imagen-país.

En este sentido, algo tan sencillo como eliminar la injustificable discriminación actual entre la fiscalidad de las becas para estos estudios en España y en el extranjero, permitiría impulsar aún más esta línea de actuación que tantos réditos aporta a España en términos de retorno científico y cultural, así como de reputación internacional.

Junto a estos aspectos, un reforzamiento general del marco regulatorio de la participación cívica y la filantropía en España redundaría, sin duda alguna, en beneficio de la acción exterior y de la imagen de España. Para ello, destacan entre las prio-

\footnotetext{
${ }^{24}$ Ejemplos de estas redes son Españoles Científicos en USA (ECUSA), que cuenta con el apoyo de la Fundación Ramón Areces, o la Spanish Global Professionals Network, apoyada por la Fundación Rafael del Pino.
} 
ridades: el fortalecimiento de la capacidad financiera de las fundaciones, mediante una mejora de su regulación ${ }^{25}$ y del tratamiento de la figura del donante; así como la reforma de la fiscalidad y de la reglamentación administrativa con el objetivo de eliminar barreras para la puesta en marcha de una fundación, pero también para el desarrollo de su actividad operativa o su accesibilidad para la ciudadanía en general (visados, controles de seguridad, limitaciones a la atracción de talento del exterior, micromecenazgo de participación y de inversión - equity crowdfunding-, etc.).

Se trata de incentivar la actividad de nuevos filántropos, de disponer de fundaciones mejores, mayores y más numerosas, pero también de favorecer la participación cívica y la democratización de la actividad filantrópica y del mecenazgo. Para ello, como se ha señalado, es necesario cambiar profundamente la narrativa social sobre estas instituciones y la regulación de las mismas, así como reconocer la generosidad del donante, de aquellos ciudadanos que, libremente, deciden actuar más allá de sus obligaciones fiscales en aras del interés general.

Señalaba Santiago Ramón y Cajal que «vivimos en un país en el que el talento científico se desconoce a sí mismo». Afortunadamente, las cosas han cambiado ostensiblemente en España desde aquellos tiempos y hoy podemos afirmar que el talento científico español se conoce a sí mismo y es crecientemente reconocido internacionalmente, como lo son la cultura española y sus protagonistas. Las diplomacias científica y cultural deben ser capaces de contribuir a impulsar la innovación y la creatividad en España mediante la promoción de la actividad, el conocimiento y la reputación de nuestros artistas, intelectuales y científicos, contribuyendo así a sumar activos intangibles a nuestra imagen-país, en una combinación óptima de atributos de imagen individuales e identitarios, integrados en una sociedad global, múltiple y diversa. Las organizaciones no lucrativas ocupan un lugar relevante en la promoción y el apoyo de las diplomacias científica y cultural y pueden suponer en el futuro una fuente aun mayor de recursos y de experiencia para apoyar el avance de la ciencia y de la cultura españolas, con un impacto directo en la reputación de España en un mundo que será liderado globalmente por el talento.

\section{Referencias bibliográficas}

Anholt, S. (2003). Branding places and nations. En R. Clifton y J. Simmons, Brands and Branding (pp. 213-226). The Economist.

Anholt, S. (2005). Place Branding. Henry Stewart Publications.

Anholt, S. (2007). Competitive Identity: The New Brand Management for Nations, Cities and Regions. Basingstoke: Palgrave Macmillan.

Álvarez, J. (2019). Hacia un nuevo paradigma para la diplomacia cultural española. Análisis del Real Instituto Elcano (ARI), 8. Real Instituto Elcano.

${ }^{25}$ La obligación establecida en la regulación de las fundaciones de gastar al menos el $70 \%$ de los recursos obtenidos anualmente por las mismas en los cuatro años siguientes a su devengo, hace imposible una gestión eficaz del capital fundacional de estas instituciones y dificulta su supervivencia a largo plazo. 
Badillo, A. (2014). Las políticas públicas de acción cultural exterior de España. Estrategia Exterior Española, 19. Real Instituto Elcano.

Dafne (2019). Country profiles Spain. Recuperado de https://dafne-online.eu/countryprofiles/\#spain

Deloitte (2017). Televisión en abierto. Contribución a la sociedad española.

ERNOP (2013). Giving in Europe. The state of research on giving in 20 European countries. Recuperado de www.europeangiving.eu

Fanjul, G. (2018, 28 de marzo). Cinco cosas que aprendí leyendo un informe sobre filantropía privada para el desarrollo. El País. Recuperado de https://elpais.com/elpais/2018/03/29/3500_millones/1522307750_940820.html

Fernández, B., \& Gross D. (2018). Las Fundaciones Filantrópicas Personales y Familiares en España. Asociación Española de Fundaciones.

Gienow-Hecht, J. C. E., \& Donfried, M. C. (Eds.) (2010). Searching for a Cultural Diplomacy. Berghahn Books.

Gouwenberg, B., Karamat Ali, D., Hoolwert, B. Bekkers, R., Schuyt, T., \& Smit, J. (2015). EUFORI Study. European Foundations for Research and Innovation. European Commision.

Hankinson, G. (2007). The management of destination brands: Five guiding principles based on recent developments in corporate branding theory. Journal of Brand Management, 14(3) 240-254. http://dx.doi.org/10.1057/palgrave.bm.2550065

Helmi, J., \& Mulyanegara, R. C. (2011). A Conceptual Framework on the Relationship between Nation Brand Perception and Donation Behaviour. International Journal of Business and Management, 6(12). http://dx.doi.org/10.5539/ijbm.v6n12p36.

Jaffe, E., \& Nebenzahl, D. (2001). National Image and Competitive Advantage: The Theory and Practice of Country-of-Origin Effect. Copenhagen: Copenhagen Business School Press.

Johnson, P. (2018). Global Philanthropy Report: Perspectives on the global foundation sector. Hauser Institute for Civil Society at Harvard University.

Keillor, B. D., \& Hult, G. T. M. (1999). A five country study of national identity: implications for international marketing research and practice. International Marketing Review, 16 (1), 65-84. http://dx.doi.org/10.1108/02651339910257656

Keillor, B. D., Hult, G. T. M., Erffmeyer, R. C., \& Babakus, E. (1996). NATID: the development and application of a national identity measure for use in international marketing. Journal of International Marketing, 4 (2) 57-73.

Keohane, R. O., \& Nye, J. (1972). Transnational relations and world politics. Cambridge, MA: Harvard University Press.

Kotler, P., Haider, D. H., \& Rein, I. (1993). Marketing Places: Attracting Investment, Industry, and Tourism to Cities, States, and Nations. New York: The Free Press.

L'Etang, J. (2009). Public relations and diplomacy in a globalized world: an issue of public communication. American Behavioral Scientist, 53 (4), 607-626. http://dx.doi. org/10.1177/0002764209347633

Marco, E., \& Otero, J. (2010). La transformación de la diplomacia cultural española. Política Exterior, 134.

Martin, I. M., \& Eroglu, S. (1993). Measuring a multi-dimensional construct: country image. Journal of Business Research, 28 (3), 191-210. https://doi.org/10.1016/0148-2963(93)90047-S 
Lenczowski, J. (2009). Cultural Diplomacy, Political Influence, and Integrated Strategy. En M. J. Waller (Ed.), Strategic Influence: Public Diplomacy, Counterpropaganda, and Political Warfare (pp.74-99). Institute of World Politics Press.

Menéndez, M. E. (2018). Diplomacia cultural: aproximación al concepto, y apuntes sobre el modelo de diplomacia cultural en España. Culturas. Revista de Gestión Cultural, 5 (2), 29-48. https://doi.org/10.4995/cs.2018.10816

Ministerio de Cultura y Deporte (2018). Anuario de Estadísticas Culturales 2018.

Nye, J. (2004). Soft power: the means to success in world politics. Public Affairs, May-June 2004.

Nye, J. (2008). Public diplomacy and soft power. The ANNALS of the American Academy of Political and Social Science, 616(1), 94-109. http://dx.doi.org/10.1177/0002716207311699

OCDE (2013). La Estrategia de Innovación de la OCDE: Empezar hoy el mañana. Foro Consultivo Científico y Tecnológico. https://doi.org/10.1787/9789264080836-es

OCDE (2017). Estudios económicos de la OCDE: España 2017. http://dx.doi.org/10.1787/9789264271920-es

Parameswaran, R., \& Pisharodi, R. M. (1992). Confirmatory factor analysis of a countryof-origin scale: initial results. Advances in Consumer Research, 19, 706-714.

Parameswaran, R., \& Yaprak, A. (1987). A cross-national comparison of consumer research measures. Journal of International Business Studies, 18 (1), 35-49. http://dx.doi. org/10.1057/palgrave.jibs.8490398

Plavsak, K. (2002). Communicative diplomacy for the 3rd millennium. Journal of Political Marketing, 1(2). http://dx.doi.org/10.1300/J199v01n02_08

Pritchard, A., \& Morgan, N. (1998). Mood marketing-the new destination branding strategy: a case study of "Wales", the brand. Journal of Vacation Marketing , 4 (3), 215-229.

Rey, M., \& y Álvarez, L. I. (2015). EUFORI Study. Spain Country Report. European Foundations for Research and Innovation. European Commission.

Reputation Institute (2018). La reputación de España en el mundo 2018. Country RepTrak

Rifkin, J. (2011). La Tercera Revolución Industrial: Cómo el poder lateral está transformando la energía, la economía y el mundo. Paidós Estado y Sociedad. Barcelona: Paidós

Rifkin, J. (2014). La sociedad de coste marginal cero: El Internet de las cosas, el procomún colaborativo y el eclipse del capitalismo. Paidós Estado y Sociedad. Barcelona: Paidós.

Roth, M. S., \& Romeo, J. B. (1992). Matching product category and country image perceptions: a framework for managing country-of-origin effects. Journal of International Business Studies, 23(3) 477-497. http://dx.doi.org/10.1057/palgrave.jibs.8490276

Royal Society (2010) New frontiers in science diplomacy. Navigating the changing balance of power, January. The Royal Society.

Rubio J.A., \& Villarroya A. (2019). El papel del mecenazgo de la política cultural española. Propuestas para reconfigurar su papel en la crisis en las artes y la industria cultural. Fundación Alternativas.

Sevin, E. (2015). Pathways of connection: An analytical approach to the impacts of public diplomacy. Public Relations Review 41 (4), 562-568.

Shimp, T. A., Saeed, S., \& Madden, T. J. (1993). Countries and their products: a cognitive structure perspective. Journal of the Academy of Marketing Science, 21(4), 323-330. http://dx.doi.org/10.1007/BF02894524 
Whetten D. A., \& Mackey A. (2002). A social actor conception of organizational identity and its implications for the study of organizational reputation. Business \& Society, 41 (4), 393-414.

Yun, S.-H., \& Toth, E. (2009). Future sociological public diplomacy and the role of public relations: evolution of public diplomacy. American Behavioral Scientist, 53 (4), 493-503. http://doi.org/10.1177/0002764209347626 